MATEC Web of Conferences 39, 01006 (2016)

DOI: $10.1051 /$ matecconf/20163901006

(c) Owned by the authors, published by EDP Sciences, 2016

\title{
Synthesis of Carbon Nanotubes and Volatile Organic Compounds Detection
}

\author{
S. Sobri ${ }^{1,2}$, J. Jasni ${ }^{1}$, Faizah M.Yasin ${ }^{1}$, Siti Hasnawati Jamal ${ }^{3}$, Nurjahirah Janudin ${ }^{3}$, and Noor Azilah Mohd Kasim ${ }^{3}$ \\ ${ }^{1}$ Faculty of Engineering, Universiti Putra Malaysia, Serdang, Malaysia \\ ${ }^{2}$ Institute of Advanced Technology, Universiti Putra Malaysia, Serdang, Malaysia \\ ${ }^{3}$ Universiti Pertahanan Nasional Malaysia, Kem Sg. Besi, Kuala Lumpur, Malaysia
}

\begin{abstract}
In this work, the adsorption effect of volatile organic compounds (chloroacetophenone, acetonitrile and hexane) towards the change of resistance of CNTs pellet as sensor signal was investigated. CNTs used in this research were synthesized using Floating Catalyst - Chemical Vapor Deposition (FC-CVD) method in optimum condition. The synthesized CNTs were characterized using Scanning Electron Microscopy (SEM), Transmission Electron Microscopy (TEM) and Raman Spectroscopy. The variation of resistance changes towards the tested gases were recorded using a multimeter. CNTs sensor pellet showed good responses towards the tested gases, however, the sensitivity, response time and recovery time of sensor pellet need to be optimized.
\end{abstract}

\section{Introduction}

Carbon nanotubes (CNTs) are globally being considered as an extremely versatile material due to its high elasticity, high conductivity, and small in size but stable and quite robust in most chemically harsh environments. Because of their extraordinary properties, CNTs have enormous potential applications in different fields such as carbon nanotubes-gas sensor[1], sensors using pristine CNTs [2], and interdigitated electrode by using CNTsbased sensor array [3].

Volatile organic compounds (VOCs) are emitted as gases from certain solids or liquids. VOCs include a variety of chemicals, some of which may have short- and long-term adverse health effects. Concentrations of many VOCs are consistently higher indoors (up to ten times higher) than outdoors. In chemical warfare, some of VOCs such as chloroacetophenone, is one of the active ingredients of Mace, a riot control agent. The advantages of CNTs such as smaller in size, high sensitivity, good response time and have capability sense various of gases, make the CNTs one of the promising material for chemical warfare agents sensor.

They can be deployed in unmanned defense systems such as unmanned aerial vehicles [4]. These electronic devices can sense the vapors emitted by explosives and chemical weapons. They are smaller in size, portable and less expensive than training bomb-sniffing dogs, which are presently used in airport, border and maritime ports. Due to this concern, investigation of gas sensing capability of CNTs towards acetonitrile, chloroacetophenone and hexane necessary as CNTs have huge potential as chemical warfare agent sensor.
In this paper, CNTs were synthesized using Floating Catalyst-Chemical Vapour Deposition method in optimum condition. The synthesized CNTs were then characterized using SEM, TEM and Raman Spectroscopy. CNTs pellet was used as sensor for detection of volatile organic compounds (VOCs) namely chloroacetophenone, hexane and acetonitrile.

\section{Methodologies}

\subsection{Synthesis of Carbon Nanotubes}

In this research, high quality of benzene $\left(\mathrm{C}_{6} \mathrm{H}_{6} 99 \%\right.$ purity, $\mathrm{R} \& \mathrm{M}$ Chemicals) was used as a hydrocarbon source and ferrocene was used as catalyst precursor. In addition, pure hydrogen and argon were used as carrier and purging gas. In a typical process of FC-CVD method, the synthesis of CNTs was carried out in a double stage furnace [5].

1 ceramic boat contained ferrocene catalyst was placed inside furnace 1 and 3 ceramic boats were placed inside furnace 2 before reaction. Initially, argon gas at a rate of $300 \mathrm{ml} / \mathrm{min}$ was flush into the CVD while raising the furnace's temperature to $900^{\circ} \mathrm{C}$.

After 1 hour, argon's flow was stopped and the 1st furnace's temperature was heat up to $150^{\circ} \mathrm{C}$ while the $2 \mathrm{nd}$ furnace's temperature was maintained at $900^{\circ} \mathrm{C}$. Then, hydrogen gas was bubble into benzene and the reaction completed after 45 minutes. CNTs synthesized were collected after the apparatus cooled down naturally.

\footnotetext{
${ }^{\mathrm{a}}$ Corresponding author: drazilahkasim@gmail.com
} 


\subsection{Characterization of Carbon Nanotubes}

Synthesized CNTs were characterized using 3 method; Scanning Electron Microscopy (SEM), Transmission Electron Microscopy (TEM) and Raman Spectroscopy. SEM was used to study the morphology of CNTs synthesized, while TEM was chosen to determine the diameter and the types of CNTs obtained. Raman spectroscopy was used to investigate the crystallinity of CNTs. In this study, the characterization was performed using a Raman spectroscopy with argon ion laser excitation at $514.532 \mathrm{~nm}$.

\subsection{Gas Sensor Set Up}

\subsubsection{Fabrication of Sensor Pellet}

For gas sensor set up, three different gases were used which are acetonitrile, n-Hexane and 2chloroacetophenone. The pellet was prepared using synthesized CNTs, ethyl cellulose and ethanol. Silver paint was used to make contact between pellet and copper wires. Last but not least, pure argon gas was used as carrier gas. 3 CNTs pellet sensor labelled as S1, S2, and S3 was fabricated by using CNTs synthesized at optimum condition. $100 \mathrm{mg}$ of synthesized CNTs were mixed with $1 \mathrm{~g}$ of ethyl cellulose as a non-conductive binder. Then, the mixture was dissolved in ethyl alcohol and was left at room temperature for 1 hour.

\subsubsection{Sensing Experimental Set Up}

Sensor pellet and three types of VOCs were employed for gas sensing applications. Electrical contact was made by connecting two copper wires with CNTs pellet using silver paint. Then, the copper wires were connected to the digital multimeter which is used to obtain and monitor the values of electrical resistance of pellet. Gas sensing experiments were carried out by placing CNTs pellet on a quartz substrate in a sealed plexiglass test chamber. Tested VOCs are filled in the sample bottle and connected to the plexiglass test chamber with an inlet valve that is used to control the injection of tested gases.

At first, the chamber was vacuumed to ensure that there are no other gases. Then, the chamber was purged continuously with pure argon until the system reached steady state. Then, the tested VOCs was injected for 1 min followed by argon gas for $5 \mathrm{~min}$. The resistance of CNTs pellet was measured before and after injection of tested gas with a digital multimeter. The experiment was repeated three times to confirm that the result were reproducible. All the measurements were taken at room temperature, $25^{\circ} \mathrm{C}$.

\section{Results and Discussion}

\subsection{Characterization of CNTs}

Figure 1 shows the CNTs synthesized at $900^{\circ} \mathrm{C}$ of reaction temperature, hydrogen flow rate $300 \mathrm{ml} / \mathrm{min}$ and 45 min of reaction time. SEM image showed that CNTs produced are hairy randomly oriented and in some cases entangled with uniform diameter with some of amorphous carbon. 45 minutes of reaction time is sufficient to produce high yield of CNTs. This implied that at longer reaction time; the presence of excess amount of carbon species would extremely enhance the formation of CNTs. As the reaction time increased, more benzene was flown into the system thus contributing more carbon source around the catalyst nanoparticles. These catalyst nanoparticles were deposited on the surface of furnace and formed catalytic layer, which later act as nucleation site for growth of CNTs [7].

High hydrogen flow rate are necessary to increase the production of CNTs [8]. Since the hydrogen was bubbled through the benzene in this study, the high flow rate of hydrogen would ultimately increase the amount of benzene carried into the system. More benzene simply implied more carbon source and finally more carbon products [8]. Other than that, the role of hydrogen as an etching agent was to remove amorphous carbon in the formation of CNTs [5].The presence of amorphous carbon was extensively minimized at this reaction time (45 $\mathrm{min})$, indicating the effectiveness of hydrogen in etching away impurities at these conditions [9].

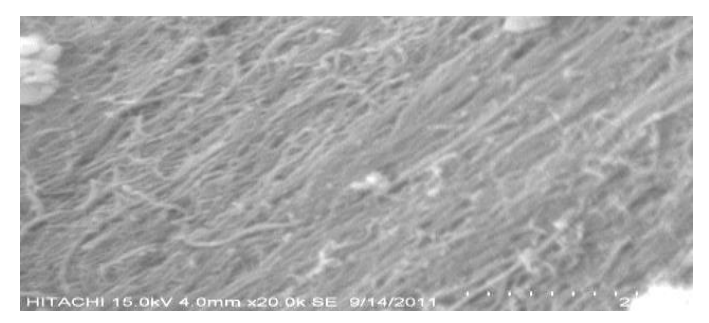

Figure 1. SEM image of CNTs synthesized at optimum condition

TEM observation was performed to enable the microstructure analysis of synthesized CNTs at optimum condition. Figure 2 shows TEM images of the CNTs synthesized at optimum conditions. The image indicated the presence of multi walled-carbon nanotubes (MWCNTs) type. Also, the images showed that the grown CNTs are long and uniform with diameter of about 8-30 nm. They exhibited exclusively tubular structure with centrally hollow inside, not a solid fiber. The lack of fringes inside the nanotube walls is evidence that the core of structure is hollow. As a result using a relatively high flow rate of hydrogen, some iron nanoparticles were driven away from the furnace, decreasing the number of catalyst particles inside the furnace, resulting in low collisions between the particles, which lead to the formation of CNTs with rather narrow diameter. The walls of these tubes were generally clean and free from amorphous coating. This indicated that amorphous carbon was only formed on the outer surface of the bundles, not between the adjacent tubes. Amorphous carbon could have vanished during ultra-sonic process for TEM imaging preparations [7] 


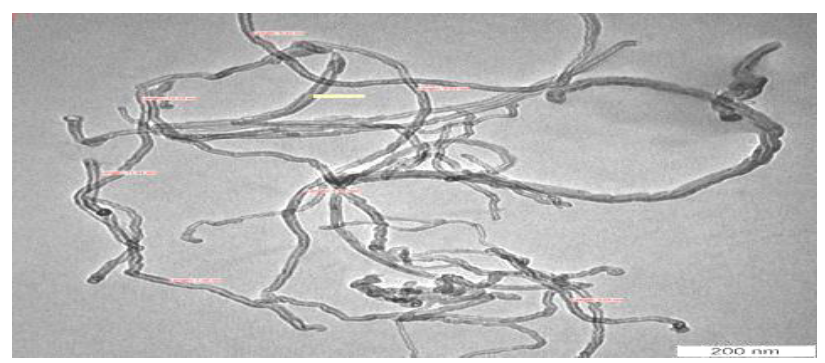

Figure 2. TEM image of CNTs synthesized at optimum condition

Lastly, the CNTs synthesized at optimum conditions were characterized by Raman spectroscopy. The Raman spectra of CNTs synthesized at optimum condition was shown in Figure 3 features in range $100-3000 \mathrm{~cm}^{-1}$, consist of two standard characteristic bands, namely the $\mathrm{D}$ band at $1742 \mathrm{~cm}^{-1}$ and the $\mathrm{G}$ band at $2227 \mathrm{~cm}^{-1}$. The D band indicates the presence of the disordered carbon structure of nanotubes and the $\mathrm{G}$ band known as graphitic mode is observed due to stretching vibration of any pair of $\mathrm{sp}^{2}$ sites [10]. The nonexistence of radial breathing mode at wavenumbers $100-200 \mathrm{~cm}^{-1}$ indicates that the presence of SWCNTs can be ruled out, and therefore, the product of the synthesized material is dominated by MWCNTs. The intensity ratio of $D$ band to $G$ band $\left(\mathrm{I}_{\mathrm{D}} / \mathrm{I}_{\mathrm{G}}\right)$ is related to degree of disorder of the nanotubes and is inversely proportional to the crystalline nature of the CNTs. In Figure 3 showed that the intensity ratio is lower, consequently higher quantity of structural defects due to its multiple graphite layer. The weak intensity of D band indicated that the CNTs synthesized were less amorphous carbon. From this result, it was confirmed that the optimum conditions of synthesized CNTs would produce the high quality of CNTs.

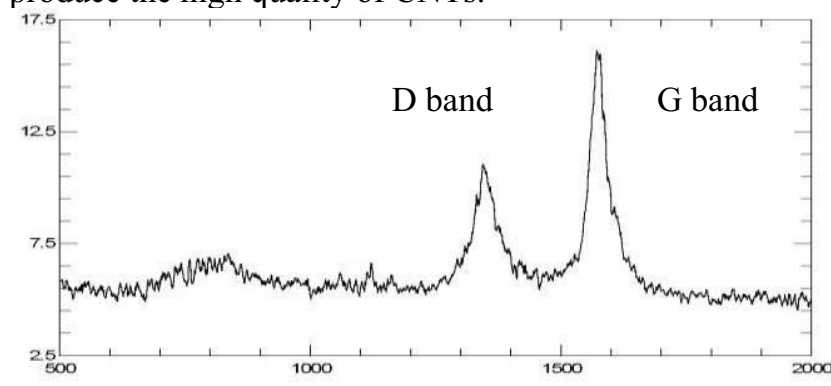

Figure 3. Raman spectra of CNTs synthesized at optimum condition

\subsection{Preliminary Study of CNTs on Gas Sensing Capability}

\subsubsection{Acetonitrile Detection}

This study was carried out to monitor and investigate the VOCs sensing effects on carbon nanotubes by a systematic study of the variations in the electrical resistance as sensor signal induced by adsorption of VOCs. The data taken were in the form of changes in resistance of CNTs vs time when the tested gas was injected or discharged at room temperature. Figure 4 shows the elecrical variation upon adsorption of acetonitrile. The constant reading for the first 60 min was due to the resistance of pellet when argon gas is purged into the system. The $3 \mathrm{CNTs}$ pellet gas sensor labelled as $\mathrm{S} 1, \mathrm{~S} 2$ and $\mathrm{S} 3$ started to show response towards acetonitrile after 14th, 7th and 17th injection; (labelled as yellow dot in Fig. 4) respectively. This proved that the CNTs pellet managed to detect tested gas from the beginning although it's not stable due to unsaturated of tested gas in the test chamber. The resistance becomes pronounced after several injections. Acetonitrile is electron donating vapour, so it's could donate electron to the valance band of CNTs, and valance band to move away from Fermi level and resulting increment of electrical resistance. However, due to the absence of heater in the sensor set up, which could increase the sensitivity of the sensor, the detection of acetonitrile takes a longer time and the recovery process is incomplete.

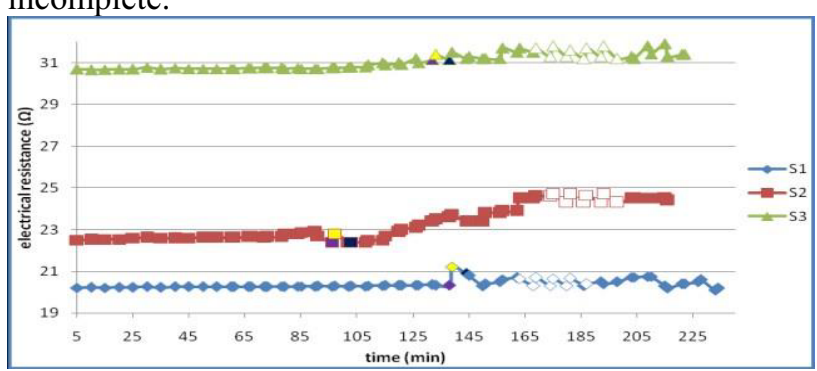

Figure 4. Electrical Resistance Variations of S1, S2 and S3 upon injection of acetonitrile

\subsubsection{Chloroacetophenone Detection}

2-chloroacetophenone, $\mathrm{C}_{6} \mathrm{H}_{5} \mathrm{COCH}_{2} \mathrm{Cl}$ also known as 2chloro-1-phenylethanone was used as tested gas. Figure 5 shows the electrical variation upon adsorption of chloroacetophenone. 3 CNTs pellet labelled as S1, S2 and S3 started to show response towards chloroacetophenone after 14th, 4th and 13th injection (labelled as yellow dot in Fig. 5), respectively. This proved that the CNTs pellet managed to detect tested gas earlier with increment of electrical resistance about $0.4 \Omega$ approximately. When CNTs are exposed to chloroacetophenone, the lone pair electron of chlorine atom transferred to CNTs, resulting the positive hole carrier of p-type semiconductor depleted and increasing of electrical resistance. The longer time taken to be detected might be because one of the main mechanisms for electrons to be excited to the conduction band is due to thermal energy; the conductivity of semiconductors is strongly dependent on the temperature of the material. In this case, chloroacetophenone did not undergo any heating treatment, resulting in slow response time of the sensor.

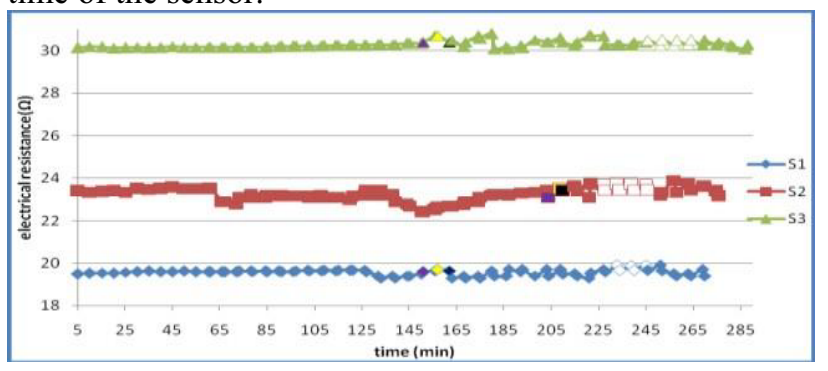

Figure 5. Electrical Resistance Variations of S1, S2 and S3 upon injection of chloroacetophenone 


\subsubsection{Hexane Detection}

After purging with argon gas, the same sensors were used to detect last tested gas, hexane. Figure 6 shows the electrical variation upon adsorption of hexane. 3 CNTs sensor pellet labeled as S1, S2 and S3 started to show response towards hexane after 17th, 27th and 16th injection (labelled as yellow dot in Fig. 6), respectively. The resistance becomes pronounced after several injections for every sensor pellet with increment $0.3 \Omega$ approximately. From the graph, it showed that hexane is poorly sensitive compared to the other two gases. Even though, hexane is volatile organic compound but with high vapor pressure, it was so difficult to react with CNTs, thus takes longer time to detect. Moreover, the sensor set up was not having any further reaction such as UV illumination, for the recovery process. It is possible that the tested gas was not removed completely and may affect the sensitivity of pellet.

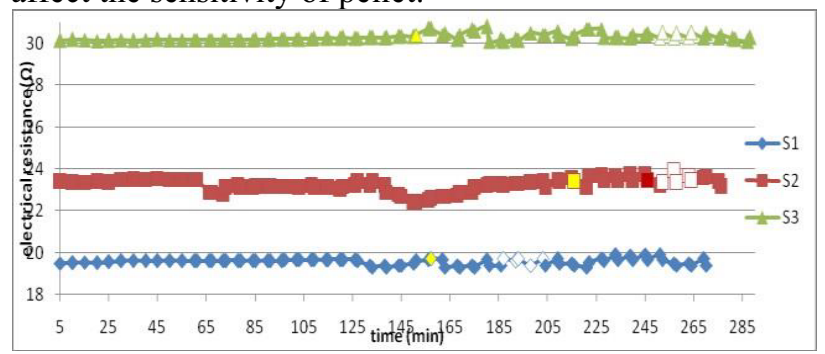

Figure 6. Electrical Resistance Variations of S1, S2 and S3 upon injection of hexane

\section{Conclusions}

The aim of this study was to investigate the gas sensing capability of CNTs pellet upon adsorption of VOCs. High quality of carbon nanotubes have been successfully synthesized via FC-CVD method using benzene and ferrocene as hydrocarbon source and catalyst, respectively. At optimum condition $\left(\mathrm{T}-900^{\circ} \mathrm{C}, \mathrm{H}_{2}\right.$ flow rate- $300 \mathrm{ml} / \mathrm{min}$, reaction time- $45 \mathrm{~min}$ ) and employing benzene as carbon source resulted in the formation high density of uniform and well-aligned CNTs having diameter in the range of 8-30 nm, with some of CNTs entangled with impurities amorphous carbon. Then, the CNTs patterned on quartz substrate were used to fabricate VOCs detectors because of the high surface area, which enable CNTs to adsorb volatile molecules easily. Based on the experimental results, the CNTs have the capability to detect acetonitrile, chloroacetophenone and hexane at room temperature and the increment of electrical resistance of sensors after VOCs exposure, due to hole depletion of p-type semiconductor CNTs. The CNTs pellet needs to overcome some weaknesses such as recovery process and sensitivity before being employed in a real situation as VOCs sensor.

\section{References}

1. Lu.Y., Partridge. C., Meyyappan. M. and Li. J. A Carbon Nanotube Sensor Array for Sensitive Gas Discrimination using Principal Component Analysis.
Journal Electroanalytical Chemistry 593 pp. 105-110 (2006)

2. Li. J. and Ang. H. T. Carbon Nanotubes Sensors. Encyclopedia of Nanoscience and Nanotechnology vol1 pp.591-601 (2004)

3. Li. J., Lu. Y., Ye.Q., Delzeit.L. and Meyyappan.M. A Gas Sensor Array using Carbon Nanotubes and Microfabrication Technology. Electrochemical and Solid State Letters 8(11), pp. 100-102 (2005)

4. Sinha. N., Ma. J. and John.Yeow.T.W. Carbon Nanotube-Based Sensors. Journal of Nanoscience and Nanotechnology 6 pp. 573-590 (2006)

5. Izawati.R.N., Fakhru'l-Razi. A., Atieh. M.A., Rashid. S. and Ali.S. Effect on hydrogen flow rate on CNT structure synthesized by CVD method. 14th Annual Scientific Malaysia Conference. (2005)

6. Mohamed.A.R. Carbon Nanotubes: The Nano material for tomorrow. Universiti Sains Malaysia, Penang, Malaysia. (2011)

7. Muhammad. S.S., Maryam. M. Syazwan. A.M.Z., Noor, A.A., Suriani. A.B., Saifollah. A., Syed Yusainee. S.Y. and Mohamad.R.M. Synthesis and Nucleation Growth Mechanism of almost-catalyst free carbon nanotubes grown from $\mathrm{Fe}$-filledsphere like graphene-shell surface. Jurnal of Nanaostructure in Chemistry.3:13. (2013)

8. Uoo-Chang.C. Effect of $\mathrm{H}_{2}$ on Formation Behaviour of Carbon nanotubes. Bull. Korean Chem Soc.Vol.25:10 pp.1521-1524 (2004)

9. Wahed, W., Kazunori, Peter, T.A.R. and Kozo, S. Experimental Characterization of the role of hydrogen in CVD synthesis of CNTs. University of Kentucky. pp.1-19 (2006)

10. Noor. A. A., Muhammad. S.S., Suriani. A.B., Mohamad. R.M. and Saifollah.A. Effect of the ratio of catalyst to carbon source on the growth of vertically aligned carbon nanotubes on nanostructured porous silicon templates.International Journal of Chemistry. 4:23-30 (2013)

11. Firouzi. A., Sobri. S., Yasin. F. M. and Ahmadun. F.R. Synthesis of Carbon Nanotubes by Chemical Vapor Deposition and Their Application for $\mathrm{CO}_{2}$ and $\mathrm{CH}_{4}$ Detection. 2010 International Conference on Nanotechnology and Biosensors IPCBEE. Vol. 2 (2011) 